\title{
Le projet d'installation laser mégajoule (LMJ) du CEA-DAM
}

\author{
D. Schirmann et M. André
}

Département projets, ingénierie et informatique, Maîtrise d'Oeuvre Principale du LMJ, CEA, BP. 12, 91680 Bruyères-le-Châtel, France

\begin{abstract}
Résumé : Afin de disposer d'un outil de simulation permettant d'étudier en laboratoire la physique des plasmas de très haute densité d'énergie, le CEADDAM envisage de construire un laser à verre dopé au néodyme de très grande puissance capable de délivrer un énergie de $1,8 \mathrm{MJ}$ en quelques nanosecondes à la longueur d'onde de $0,35 \mu \mathrm{m}$. Cette énergie correspond à l'énergie minimale nécessaire pour obtenir la combustion d'une petite quantité de deutérium et de tritium avec un gain significatif (environ 10 fois l'énergie laser investie). L'article décrit l'installation et les problèmes de conception de la chambre d'expériences liés à l'énergie libérée lors de la combustion du DT.
\end{abstract}

\section{INTRODUCTION}

L'étude de plasmas denses et chauds tels que ceux créés par interaction laser matière a conduit le CEAJDAM à développer la technologie des lasers de puissance à verre dopé au néodyme. Le laser Phébus fonctionnant au CEA - Limeil Valenton délivre après conversion de fréquence une énergie de $8 \mathrm{~kJ}$ en une nanoseconde (ns) à la longueur d'onde de $0,35 \mu \mathrm{m}$. En implosant, suivant un schéma d'attaque indirecte, des cibles sphériques contenant un mélange équimolaire de deutérium (D) et de tritium (T), ce laser a permis (1) en particulier de comprimer du DT à environ 100 fois sa densité à l'état solide $\rho_{0}$ et de le chauffer à une température $T$ d'environ $1 \mathrm{keV}$. Les codes de simulation numériques de ces expériences montrent qu'avec un laser plus puissant, il serait possible d'atteindre les conditions de température $(T>20 \mathrm{keV})$, de densité $\left(>1000 \rho_{0}\right)$ et de temps de confinement $(100 \mathrm{ps})$ permettant de franchir le seuil de l'allumage et d'obtenir la fusion du DT.

L'installation Laser Mégajoule a été dimensionnée pour obtenir l'allumage et la combustion (2) d'une petite quantité de DT en laboratoire avec un gain d'environ 10 défini par le rapport entre l'énergie de fusion produite et l'énergie laser apportée. Des simulations numériques effectuées avec les codes d'implosion FCI à la DAM et Lasnex au LLNL sur des cibles représentatives montrent que l'énergie laser minimale nécessaire est d'environ $1,8 \mathrm{MJ}$ et doit être délivrée en quelques ns. Ces simulations ont permis de dimensionner les expériences et de définir les spécifications techniques de base à la fois des cibles et du laser. Ce article donne les principales caractéristiques de l'installation (3).

\section{LA CIBLE}

La cible type permettant d'atteindre l'allumage et un gain de 10 est une cible dite cryogénique représentée sur la Fig. 1. Elle constituée d'un microballon en plastique de $2 \mathrm{~mm}$ de diamètre, de $200 \mu \mathrm{m}$ d'épaisseur de paroi contenant sur sa paroi intérieure une couche de DT gelé de $200 \mu \mathrm{m}$ d'épaisseur. Le microballon est placé dans une cavité en or de $5 \mathrm{~mm}$ de diamètre et de 
7 mun de longureur munie à ses extrémités de 2 trous de $1,5 \mathrm{~mm}$ de diamètre permettant le passage des faisceaux laser. La cible fonctionne selon un schéma d'attaque indirecte étudié avec le laser Phébus. Lors de l'interaction avec la paroi intérieure de la cavité, les faisceaux laser sont convertis en rayonnement $\mathrm{x}$. La pression exercée par les rayons $\mathrm{x}$ implose le microballon, comprimant et chauffant le DT jusqu"à l'obtention des conditions d'allumage et de combustion. Les dimensions de la cible et sa structure ont été optimisées pour atteindre l'objectif avec l'énergie laser minimale.
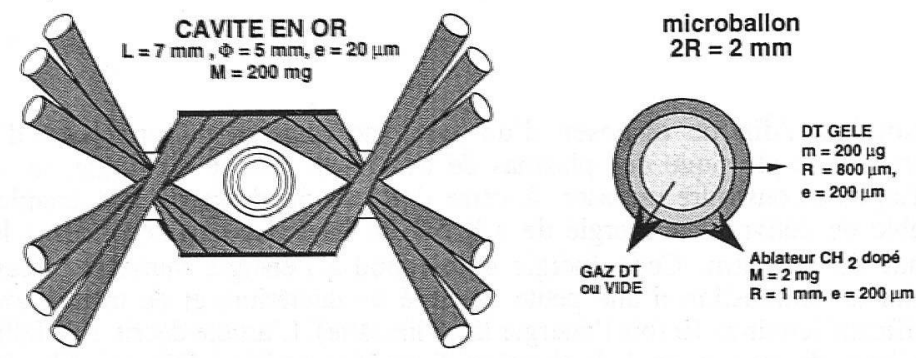

Fìg. 1 : Schéma de la cible cryogénique permettant d'obtenir l'allumage du DT par point chaud et sa combustion

\section{I.E LASER}

Les specificiations de base du laser sont donnees dans le tableau I. Parmi les moyens potentiellement capables de répondre à une telle spécification, le laser à verre dopé au néodyme est apparu comme le meilleur candidat pour des raisons de coût de développement et de maîtrise des technologies. En particulier, les progrès réalisés depuis 10 ans ont permis de diminuer le coût du joulte laser de pluss d'un ordue de grandeur par rapport à Phébus.

Tableau I : Spécifications techniques du laser LMJ

\begin{tabular}{|c|c|}
\hline 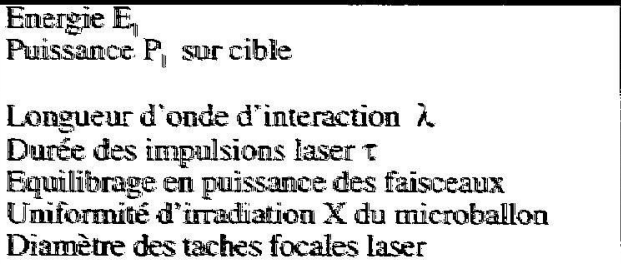 & $\begin{array}{c}>1,8 \mathrm{MJ} \\
\text { montée progressive par paliers jusqu'à } \\
\mathrm{P}_{\mathrm{t}}>600 \mathrm{TW} \\
0,35 \mu \mathrm{m} \\
17 \mathrm{~ns} \\
<7 \% \text { rms par tranches de } 2 \mathrm{~ns} \\
<2 \% \mathrm{~ms} \\
<500 \mu \mathrm{m}\end{array}$ \\
\hline $\begin{array}{l}\text { Prócision de pointage des fainsceaux sur la cible } \\
\text { Cadence die tirs }\end{array}$ & $\begin{array}{c}50 \mu \mathrm{m} \\
>600 \text { tirs par an dont } 12 \text { tirs par dégageant une } \\
\text { énergie de } 20 \mathrm{MJ}\end{array}$ \\
\hline
\end{tabular}

Pour répondre à ces spécitications, le taser est constitué de 240 faisceaux unitaires de section carrée de $40 \mathrm{~cm}$ de côté. Le dimensionnement des chaines laser prend en compte l'ensemble des limitations physiques connues aujourd'hui, comme les effets de saturation du gain laser et de l"anplificiation spontamée dans les disques, la tenue au flux laser des composants optiques, les effets des indices nonlinêaires des matểriaux sous le champélectrique de l'onde laser, les pertes résultant des diffusions transverses non linéaires comme celles dues à la diffusion Raman et Brillouin. les pertes liêes aux biréfringences induites dans les optiques soumises à des 
contraintes thermiques ou mécaniques, les incidences sur la qualité de la surface d'onde et de la tache focale des aberrations créées par les défauts de planéité des optiques... Tous les phénomènes ont été modélisés et rassemblés dans un ensemble de codes de simulation appelé MIRO validé à partir des résultats acquis sur le laser Phébus permettant d'optimiser les composants entrant dans le schéma de base du LMJ, tant en terme de spécifications techniques qu'en terme de coût de fabrication.

Le schéma de base d'une chaîne amplificatrice est représenté sur la Fig. 2. Pour réduire les coûts de fabrication, tous les composants sont de mêmes dimensions. Chaque faisceau unitaire est constitué d'une cavité amplificatrice et d'un filtrage spatial de transport assurant l'injection et l'extraction du faisceau. L'impulsion provient d'un pilote où elle est créée et mise en forme

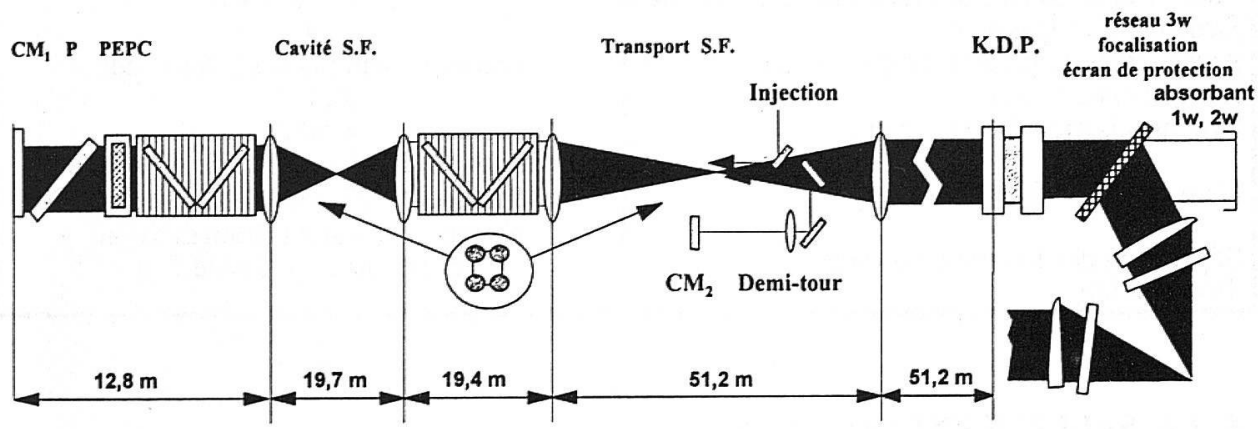

Fig. 2 : Schéma de base de la structure d'un faisceau élémentaire : $\mathrm{CM}_{1}$ et $\mathrm{CM}_{2}$ sont les miroirs de fond de cavité, $\mathrm{P}$ est un polariseur, PEPC une cellule de Pockels et $\mathrm{S}$. F. sont des filtrages spatiaux.

temporellement. Elle est transportée par fibre optique jusqu'à une unité de préamplification située sur le filtrage de transport. La cavité est constituée, entre 2 miroirs, de 2 ensembles amplificateurs identiques de 9 disques chacun et d'un filtrage spatial. Le rendement d'extraction de l'énergie laser est augmenté grâce à 4 passages du faisceau dans la cavité amplificatrice permis par un jeu de miroirs appelé « demi tour». Le miroir de cavité est un composant devant avoir une très haute tenue au flux laser, dont la technologie est acquise. Une cellule de Pockels et un polariseur assurent la coupure de l'émission spontanée.

Le rendement du laser est également augmenté en adoptant une structure multi segmentée qui améliore le couplage optique entre les lampes flashes et les disques laser. Les faisceaux unitaires sont regroupés par paquets de 8 ( 4 faisceaux en hauteur et 2 faisceaux en latéral) pour créer une unité amplificatrice de $4 \times 2$ faisceaux. A la sortie de ces unités, les faisceaux unitaires sont regroupés par 4 et transportés par un jeu de miroirs successifs vers les cristaux de conversion de fréquence et la chambre d'expériences.

La changement de longueur d'onde du laser de $1,05 \mu \mathrm{m}$ à $0,35 \mu \mathrm{m}$ s'opère dans un ensemble de 2 cristaux de KDP de $40 \mathrm{~cm} \times 40 \mathrm{~cm} \times 1 \mathrm{~cm}$ avec un rendement de 55 à $70 \%$ suivant les conditions du flux laser incident. Ils font l'objet d'un effort de développement très important pour en augmenter la vitesse de croissance tout en conseryant une bonne homogénéité.

Un réseau de diffraction par transmission fonctionnant à $0,35 \mu \mathrm{m}$, est utilisé pour séparer les longueurs d'onde non converties 1,05 et $0,53 \mu \mathrm{m}$ d'une part et pour changer la direction $\left(60^{\circ}\right)$ du faisceau à $0,35 \mu \mathrm{m}$ et éviter ainsi aux composants du laser et aux diagnostics de fin de chaîne d'être en vue directe de la cible et de l'émission neutronique. Le faisceau est ensuite focalisé par une lentille protégée des émissions de la cible par une lame de silice. Les réseaux font également l'objet d'un important effort de développement. Pour limiter les pertes par réflexion, tous les dioptres sont traités par un revêtement anti reflet du type « sol gel " dont la tenue au flux a fait de très grands progrès au cours de ces dernières années. 
Le tableau II résume le schéma de base du laser. Les coûts de recherche et développements sont partagés entre le CEA et le DOE dans le cadre d'un accord de collaboration. Un prototype d'un faisceau unitaire appelé «Beamlet » a été construit au LLNL. Il a permis de valider la structure de la chaîne et ses nouveaux concepts. Il a permis notamment en 1995 de vêrifier que l'énergie de sortie correspondait bien à l'objectif.

Tableau : II : Schéma de base du laser

Nombre de faisceaux élémentaires
Section des faisceaux
Energie et puissance des faisceaux élémentaires
Structure multipassage
Architecture de la cavité amplificatrice
Rendement du laser
Synchronisation des faisceaux
Conversion de fréquence
Séparation des longueurs d'onde
Focalisation

\section{LA SALLE D'EXPERIENCES}

La salle d'expériences et ses équipements sont une extrapolation de ceux du laser Phébus. On y trouve une chambre d'expériences sous vide de $10 \mathrm{~m}$ de diamètre, les équipements nécessaires à la mise en place des cibles au centre de la chambre, un ensemble de miroirs de renvoi des faisceaux vers la chambre, les systèmes de conversion de fréquence, de séparation des longueurs d'onde et de focalisation, les diagnostics de fin de chaîne et les diagnostics du plasma.

Les contraintes sont cependant très nouvelles en raison du plus grand nombre de faisceaux et, lors de tirs à fort gain, de l'importance des énergies qui seront dégagées. L'énergie libérée apparaît sous la forme de rayons $x$, de particules chargées de grande vitesse appelées débris, et de neutrons de $14 \mathrm{MeV}$ résultant de la combustion thermonucléaire du DT.

A titre indicatif, pour une énergie laser de $2 \mathrm{MJ}$, l'énergie thermonucléaire libérée en une centaine de picosecondes (ps), par une cible à gain 10 serait de $22 \mathrm{MJ}$, répartis en $3 \mathrm{MJ}$ de rayons $\mathrm{x}, 3 \mathrm{MJ}$ de débris et $16 \mathrm{MJ}$ de neutrons soit près de $10^{19}$ neutrons.

L'interaction des neutrons de $14 \mathrm{MeV}$ avec les matériaux qu'ils rencontrent peuvent générer des rayons $\gamma$ prompts par réaction $(n, \gamma)$ et, par réaction de transmutation des rayons $\gamma$ de désactivation, émis pendant des temps plus ou moins longs suivant les matériaux irradiés pouvant limiter les temps d'accès à la chambre après un tir. Les émissions de neutrons et de rayons $\gamma$ entaînent la mise en place de murs de radioprotection pour assurer la sécurité du personnel et pour ce qui est de la conception de la chambre et de ses équipements, à ne retenir que des matériaux à courte période de décroissance radioactive quand c'est possible.

\subsection{Le schéma d'irradiation}

Les faisceaux laser regroupés par 4 arrivent sur la cible moitié par le haut, moitié par le bas, l'axe de la cavité étant vertical, libérant ainsi le plan horizontal pour la fonction diagnostics. Ils sont distribués suivant trois cônes concentriques de demi-angle au sommet respectivement $26^{\circ}$ 
(11 faisceaux), $45^{\circ}$ ( 11 faisceaux), $59^{\circ}$ ( 11 faisceaux). Toutefois ces angles sont susceptibles de changer. Pour avoir plus de flexibilité dans le schéma d'irradiation et en particulier pour pouvoir imploser des cibles à la fois en attaque directe et en attaque indirecte, nous recherchons d'autres configurations.

\subsection{La chambre d'expériences}

C'est une enceinte sous vide secondaire représentée sur la Fig. 3, rigide pour résister à la pression atmosphérique. Elle supporte tous les équipements (porte cible non cryogénique et cryogénique permettant de geler le DT, les pompes cryogéniques, les diagnostics du plasma) et doit permettre l'entrée des faisceaux. Elle est de forme sphérique constituée d'une double paroi en alliage d'aluminium de 25 et $20 \mathrm{~mm}$ d'épaisseur, espacées de $80 \mathrm{~cm}$ pour pouvoir y introduire un matériau neutrophage capable d'atténuer le flux de neutrons d'un facteur $10^{3}$. Le choix d'un alliage d'aluminium est justifié par la nécessité de réduire le temps d'accès à l'intérieur de la chambre à une semaine environ après un tir avec gain.
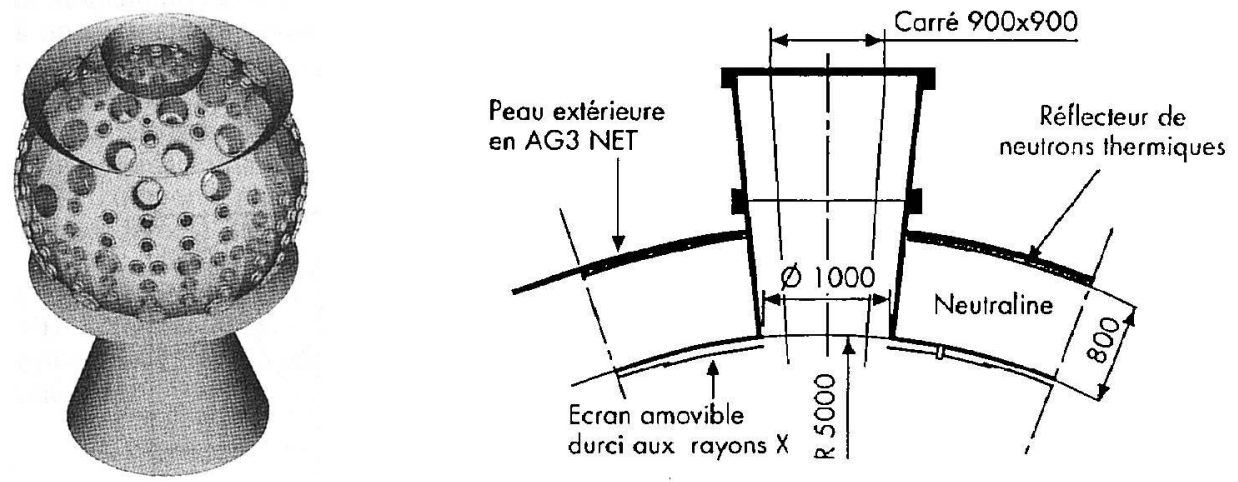

Fig. 3 : Schéma de la chambre d'expérience et de la structure de la paroi

La protection de la paroi intérieure contre les rayons $\mathrm{x}$ émis par la cible fait l'objet d'études approfondies (4) On prévoit d'utiliser des écrans de protections amovibles en matériaux plus réfractaires que l'aluminium tels que le $\mathrm{B} 4 \mathrm{C}$ ou le carbone capables de résister sans se vaporiser à des fluences $\mathrm{x}$ de l'ordre de $1 \grave{a} 2 \mathrm{~J} / \mathrm{cm}^{2}$, le spectre du rayonnement émis correspondant à un spectre de corps noir de $400 \mathrm{eV}$ de température.

\subsection{La mise en place des cibles}

La chambre sera équipée d'un porte cible à température ambiante permettant d'introduire des cibles non cryogéniques et de les positionner au centre avec une précision de l'ordre de $5 \mu \mathrm{m}$. L'introduction de cibles contenant du DT gelé dans les microballons nécessitera un porte cible dit cryogénique. Il sera muni d'un cryostat permettant de maintenir le microballon au voisinage de la température du point triple ( $19^{\circ} 8 \mathrm{~K}$ pour le DT). L'élaboration des cibles avec du DT gelé répondant aux spécifications pour obtenir l'allumage et la combustion fait l'objet d'un très gros effort d'études et de développement.

\subsection{Les diagnostics du plasma}

Les premiers diagnostics de plasma qui permettront d'accompagner la montée en puissance du laser jusqu'à la réalisation de tirs avec combustion thermonucléaire du DT ont êté définis sur la base d'un scénario expérimental comportant 2 phases : 
Phase I : elle comportera plusieurs étapes. Après avoir qualifié le laser en tirant sur des cibles planes, on étudiera la répartition spatio-temporelle des sources de rayonnement $\mathrm{x}$ créées sur la paroi intérieure de la cavité, l'hydrodynamique de l'implosion en cavité de microballons remplis de DT gazeux, l'obtention des conditions d'allumage en implosant des cibles cryogéniques dites inertes, réalisées avec du DD gelé ou des mousses remplies de DT pour conserver une cadence de tirs élevée.

Phase II : elle correspond à la phase des tirs avec gain, dans les conditions que la phase I aura permis de préciser, pour étudier la combustion du DT avec des cibles cryogéniques utilisant cette fois du DT gelé, la cadence de tirs étant limitée par les forts dégagement d'énergie dus à la combustion du DT.

La Fig 4 précise les dimensions, les temps caractéristiques et les températures que les diagnostics de plasma auront à mesurer : la cavité de l'ordre du $\mathrm{cm}$, pendant $20 \mathrm{~ns}$, dont la température de rayonnement maximale pourra atteindre $400 \mathrm{eV}$ au moment de la combustion, l'évolution spatio-temporelle du microballon de dimensions millimétriques pendant quelques nanosecondes dont la température pourra monter jusqu'à quelques $\mathrm{keV}$ avant la combustion, la zone de combustion de l'ordre $0,1 \mathrm{~mm}$ pendant $100 \mathrm{ps}$, sa température montant jusqu'à plusieurs dizaines de $\mathrm{keV}$ pendant la combustion.
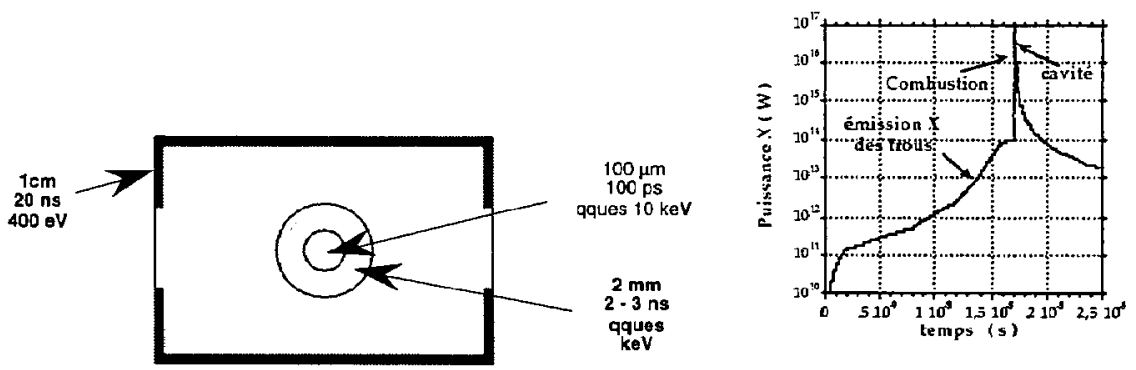

Fig. 4 : Grandeurs caractéristiques à mesurer

Fig.5 : Variation temporelle de l'émission $x$ d'une cible à gain

Les diagnostics sont principalement des diagnostics utilisant les émissions uv, $\mathrm{x}, \gamma$, et neutronique de la cible. Ils comportent pour l'essentiel des systèmes d'imagerie et de spectroscopie associés à des caméras à balayage de fente ou des tubes obturateurs analogues à ceux utilisés sur la chambre d'expériences du laser Phébus. Toutefois la faible durée de vie de la zone de combustion nécessite des résolutions spatiale et temporelle respectivement inférieures à $10 \mu \mathrm{m}$ et à 10 ps impliquant de maintenir l'effort actuel (5) sur le développement des techniques de cinématographie ultra-rapide, ainsi que dans le domaine de l'imagerie des émissions $\mathrm{x}$ dur, $\gamma$ et neutronique.

A titre d'exemple la Fig. 5 représente la variation temporelle de l'émission $\mathrm{x}$ d'une cible à gain dans une cavité mince (épaisseur $20 \mu \mathrm{m}$ ). Le pic d'émission correspond au dégagement d'énergie $\mathrm{x}$ au moment de la combustion (environ $3 \mathrm{MJ}$ de rayons $\mathrm{x}$ ). 


\section{ETAT D'AVANCEMENT ET PLANNING}

Le LMJ sera construit au CEA-CESTA en Aquitaine. La construction comporte plusieurs phases :

- construction, dès 1997, d'une ligne d'intégration laser (LIL) constituant une chaîne prototype des 30 chaînes du LMJ, soit 8 faisceaux de $40 \mathrm{~cm}$ x $40 \mathrm{~cm}$ délivrant sur cible $60 \mathrm{~kJ}$ de lumière à la longueur d'onde de $0,35 \mu \mathrm{m}$. Cette chaîne pourrait être utilisée pour faire des expériences sur cible à partir de 2001.

- après validation de la chaîne prototype, le planning prévoit de construire le LMJ en deux étapes, une première étape avec un tiers de l'installation soit 10 chaînes laser délivrant sur cible $600 \mathrm{~kJ}$ utilisable sur cible à partir de 2005 , puis la totalité de l'installation, soit 30 chaînes délivrant 1,8 MJ, utilisables sur cible à partir de 2010 .

\section{CONCLUSION}

Pour disposer de moyens de simulation permettant de poursuivre l'étude des plasmas de très haute densité d'énergie en laboratoire, le CEA DAM construit au CESTA en Aquitaine une installation laser de très grande puissance : le laser mégajoule (LMJ). Elle a été dimensionnée pour obtenir l'allumage et la combustion d'une petite masse de DT. Sa construction prévue pour 2010 comporte plusieurs étapes, la première consistant à construire une chaine prototype appelée ligne d'intégration laser, dont le l'utilisation sur cible est prévue à partir de 2001.

Le laser MJ constituera la source de rayonnement UV la plus importante au monde avec celle du NIF (National Ignition Facility) (6) en cours de construction au LLNL (USA). Ce sera également l'une des sources de rayonnement xuv et neutronique les plus puissantes jamais réalisées.

\section{Références}

(1) Fortin X., Chocs, Revue scientifique et technique de la DAM, Expériences d'implosion en attaque indirecte, 13 (1995) 13-23

(2) Bayer C. et al. Chocs, Revue scientifique et technique de la DAM, Combustion du DT et dimensionnement d'une cible à haut gain, 13 (1995) 35-45

(3) André M., Novaro M., Schirmann D., Chocs, Revue scientifique et technique de la DAM, Technologie pour un laser mégajoule, 13 (1995) 73-84

(4) Schirmann D., Tobin M., 12th topical meeting on the technology of fusion energy, Reno (NV), target conceptual design issues of the french laser megajoule facility (LMJ) (1996) à publier

(5) Schirmann D. Annales de physique 17 (1992) 109-116

(6) Energy and technology review, The National Ignition Facility, University of California, Lawrence Livermore National Laboratory, (dec. 1994) 